\title{
Nelson CHAREST
}

\section{Wagner hors de soi}

L'essai d'Éric Touya de Marenne, Musique et poétique à l'âge du symbolisme, se veut une suite de "Variations sur Wagner», comme l'indique le sous-titre. À partir des critiques de Baudelaire, Mallarmé, Claudel et Valéry, l'auteur entend analyser l'impact de l'œuvre et de la pensée du compositeur allemand sur les poètes français, sans reprendre l'analyse plus politique qu'avait déjà proposée Philippe Lacoue-Labarthe $^{1}$. L'auteur veut plutôt "expliquer le pourquoi et le comment de ces variations » et « explorer ce qui distinguait les poètes de Wagner, et à l'arrière plan, de Schopenhauer et Nietzsche $»^{2}$. C'est donc dire que l'étude se place de plain-pied dans le discours esthétique, sinon poétique, et chez Wagner et chez les poètes qui ont subi son influence. On ne trouvera donc aucune référence aux livrets ni aux partitions du compositeur; des partitions sont bien reproduites aux pages 74 et 76 , mais sans que l'auteur les commente. La même remarque s'applique aux poètes, cités surtout pour leurs essais critiques, et particulièrement ceux qui concernent directement Wagner, ce qui précise considérablement la portée de son influence.

Cet intérêt pour l'esthétique wagnérienne ne s'écarte jamais tout à fait du terrain du wagnérisme, malgré la prudence affichée en quatrième de couverture: "Afin de mesurer la portée de son œuvre, il nous fallait examiner cette dernière à la lumière du sens que lui prêtaient les poètes, et explorer les motifs d'une méditation qui, de 1860 à 1945, allait à bien des égards à contre-courant du wagnérisme. » Il faut

\footnotetext{
${ }^{1}$ Philippe Lacoue-Labarthe, Musica Ficta. Figures de Wagner, Paris, Christian Bourgeois, 1991. Touya de Marenne y réfère à la page 11, op. cit. : «Tout en reconnaissant les circonstances historiques et politiques, mais aussi la dimension philosophique, qui ponctuaient l'avènement du wagnérisme en Europe, nous considérions suivant un autre point de focalisation la portée de l'œuvre et de la pensée du compositeur. »

${ }^{2}$ Ibid.
} 
d'abord considérer que les œuvres de Wagner sont relativement peu connues à cette époque : il faudra attendre le début du $\mathrm{XX}^{\mathrm{e}}$ siècle pour que l'ensemble des opéras soient représentés en France, comme le rappelle justement l'auteur. Cela dit, l'insécurité d'un Baudelaire qui, comme le souligne régulièrement Touya de Marenne, est confronté à «l'indescriptible » de ses impressions, doit être relativisée. Baudelaire est très conscient qu'il détient le minimum d'informations pour élaborer sa critique, que le souvenir d'une soirée d'opéra n'est pas aussi fiable qu'un texte qu'on aurait sous la main, que la musique ellemême confine au non-langage. Que le poète de la modernité ait appliqué à l'œuvre wagnérienne sa propre grille esthétique surprend moins, dans ce contexte. Malgré tout, l'auteur affirme de son côté que cette disjonction marque un tournant :

Tout autant qu'une «baudelairisation » de Wagner, cette interversion mettait un terme à la prétendue supériorité du musicien. L'affirmation de Baudelaire portant sur la suprématie de sa critique et de son art était à cet égard décisive. Elle situait le musicien et l'ensemble de son œuvre dans la perspective d'une imagination créatrice, soumettant le compositeur en somme aux principes de la poétique baudelairienne. (p. 69)

Baudelaire, qui peut interpréter l'opéra « sans le livret », se voit ici posé en rival de Wagner, puisqu'il entendrait remplacer la musique par la critique d'imagination. Certes, le poète se porte à la défense de la « reine des facultés », mais il le fait précisément dans un contexte critique; il est clair, à mon avis, que les rivaux sont plutôt à chercher du côté des autres critiques, fondus dans la masse des auditeurs, ceuxlà mêmes qui ont sifflé la représentation du Tannhäuser en 1861. Face à Wagner, la véritable différence est de l'ordre du vocabulaire: si Baudelaire reconnaît chez Wagner une poétique qui lui est (déjà) propre, c'est tout simplement qu'il le traduit en un langage qu'il connait, ce qui est, au fond, la seule façon d'introduire une œuvre étrangère. On le voit ici, et cet aspect est perceptible tout au long de l'étude de Touya de Marenne: celui-ci est plus intéressé par les différences, entre les poètes eux-mêmes et avec Wagner, quitte à les forcer un peu, à ce qu'il me semble. 
Si j'insiste tant sur ce point, c'est qu'il a un impact considérable dans cette analyse, à cause justement de son développement en « variations ». Les quatre poètes étant étudiés successivement selon un ordre chronologique, autour d'un centre wagnérien (lui-même en développement, l'ensemble de l'œuvre se faisant connaître de plus en plus avec le temps, ce dont l'auteur n'a peut-être pas assez tenu compte), le développement avance par des redites et des amplifications, toujours à partir de ce qui a été dit. Étant le premier auteur traité, Baudelaire acquiert, selon moi, une importance un peu artificielle, qui ne dépend que de la structure adoptée. Certes, les poètes subséquents auront eux-mêmes pris connaissance des critiques baudelairiennes, qui ont donc une primauté dans les faits. Mais ce qui gêne ici, c'est que la critique baudelairienne n'est plus soumise, justement, à la «variation", du moment que l'auteur l'a interprétée; ce que Touya de Marenne entend comme une «transition» chez Baudelaire demeure alors telle quelle chez Mallarmé, Claudel et Valéry, sans considération de leurs lectures, à eux, du texte baudelairien. Cette méthode permet certes de mieux marquer les différences, mais d'une façon quelque peu artificielle.

Or, cette lacune même nous renseigne encore sur l'art wagnérien, qui est l'œuvre «de la philosophie et non de la poésie» (p. 259), pour reprendre les termes de Valéry. Paradoxalement, c'est bien l'auteur de l'étude qui semble avoir substitué à l'œuvre wagnérienne la critique baudelairienne, celle-là s'éclipsant de plus en plus derrière les discours seconds. Dès le départ, on l'a noté, les livrets sont évacués; et par la suite, la «Lettre sur la musique » apparaît certes souvent, mais presque seule parmi l'œuvre du compositeur. Cette lettre, qui aura incidemment servi à Baudelaire pour écrire son article, étant ellemême un discours critique, et second, l'essai lorgne du côté du wagnérisme, d'une connaissance de seconde main des opéras euxmêmes. Comme l'auteur le souligne, l'intérêt de cette étude réside surtout dans la «conception de la littérature» que les auteurs vont opposer à Wagner (p. 23). On retrouve donc ici ce glissement, tout wagnérien, entre l'œuvre elle-même et l'effet qu'elle produit.

C'est donc sur le terrain de la connaissance des auteurs français et de leurs poétiques qu'il faut replacer cet essai. Ainsi l'adage mallarméen, 
de « reprendre à la musique son bien », est-il réinterprété dans un sens abstrait et idéaliste, en tant que quête d'un langage débarrassé du concept, comme peut l'être la poésie. Ainsi également le dégagement d'un «esprit français» (p. 103-109), perceptible chez Mallarmé, Debussy et Baudelaire, et qui répondrait au "vague des passions » wagnérien par la clarté et la synthèse. Par la confrontation des systèmes, par de nouveaux regroupements, c'est l'œuvre de Mallarmé qui s'éclaire dans ces exemples et qui profite du point de vue wagnérien. Le chapitre sur Claudel, où on retrouve en leitmotiv le mot de l'Ecclésiaste, Non impedias musicam (n'empêchez pas la musique), filé et modulé tout du long, est peut-être le plus éclairant. La situation historico-géographique de Claudel, son intertexte, sa poétique (du verset, du théâtre), sa morale et ses croyances semblent trouver dans l'œuvre wagnérienne leur point de fuite, à la fois rejeté et structurant, mis hors-jeu mais, en même temps et du fait même, au revers duquel se formera la multiplicité des œuvres. C'est ici que la confrontation semble la plus énergique, en ce que, précisément, elle met face à face deux champs d'énergie opposés et qui se révèlent en contrastes. La partie sur Valéry, à l'inverse, montrerait plutôt une intégration parfaite, une appropriation sinon un détournement de "Wagner», réduit à un symbole sinon à une idole, et serait peut-être de ce fait moins instructive; on a l'impression d'y retrouver un Valéry déjà connu, où le «Wagner» est prêt de s'effacer. Mais peut-être ainsi la variation wagnérienne trouve-t-elle une structure secrète, dialectique certainement, de la reconnaissance baudelairienne à l'idéal mallarméen, de l'écho multiple claudélien à l'assimilation valéryenne. L'histoire ne dit pas le sombre héritage que connaitra Wagner par la suite, ni son insidieuse diffusion inconsciente depuis. Parions que la poésie française saura bientôt éclairer à nouveau une œuvre qui semble faite tout autant pour les réponses qu'elle suscite que pour son bien propre, toujours repris mais jamais achevé.

Référence : Éric Touya de Marenne, Musique et poétique à l'âge du symbolisme. Variations sur Wagner: Baudelaire, Mallarmé, Claudel, Valéry, Paris, L'Harmattan, coll. «Littératures comparées », 2005, $286 \mathrm{p}$. 